\title{
Fear versus Taste for the Apnea at the Schelling-Point Game in the Swimming Pool
}

\author{
Luc Collard ${ }^{1}$, Corinne Fantoni ${ }^{1}$, Alexandre Oboeuf ${ }^{1,2}$ and Élise Defrasne Ait-Said ${ }^{1}$ \\ 1. Department of Sports Science and Physical Education, University of Paris Descartes, rue Lacretelle 75015, France \\ 2. Department of Ethics, University Catholic of Lille, rue du Port 59000, France
}

\begin{abstract}
We studied a pure coordination game in which swimmers had to agree on the distance that they would swim underwater up a line after diving into a swimming pool. The swimmers were not able to communicate with each other. To win the game, the swimmer had to surface at exactly the same point as his partner. This pure coordination game was played by 81 swimmers, 43 of whom then put their decisions into practice in a 25-metre pool. The results revealed the presence of two Schelling points. One point (at 12.5 metres) was that predicted by theory and corresponded to half the maximum possible distance. The other Schelling point (at 25 metres) was practical in nature and reflected a taste for underwater swimming-even though performance (in terms of the distance swum underwater) was not relevant in this game. The results show that swimmers underestimate their water competence when they are not subjected to a competitive challenge.
\end{abstract}

Key words: Underwater swimming, free-diving, pure coordination game, schelling point, game theory.

\section{Introduction}

By studying the difference between real and perceived motor skills, one can estimate the role of the subconscious in motor action and thus understand how sportspersons might improve their performance [1-5]. The famous swim coach James Counsilman noted that as a young swimmer, Mark Spitz was unaware that his hands were making "sculling" movements during the underwater pull stroke. Although Spitz considered that his arm strokes were linear, underwater filming showed that his hands followed a sinuous path and that his elbows were even flexed at $90^{\circ} \mathrm{C}$ at some points [6]. Counsilman does not say whether this realization enabled Spitz to win his seven gold medals at the 1972 Munich Olympics.

The aquatic milieu amplifies disparities between real and perceived motor skills. Visual and proprioceptive references are significantly modified [7]. Although many researchers have shown that young men

Corresponding author: Luc Collard, Ph.D., professor, research fields: game theory and the emergence of fifth stroke. E-mail: luc.collard@parisdescartes.fr. overestimate their swimming competence more than young women do [8-12], it has not been established whether these overestimations are accurate or not. This type of scientific knowledge is nevertheless important for drowning prevention [13]. Overestimation of swimming competence can lead to underestimation of the dangers associated with the aquatic milieu [14] and/or may lead to aquaphobia $[15,16]$.

Experimentally, it is difficult to measure swimming competence without placing the participants at risk. In fact, performance tests are likely to generate reckless behavior [17] and may lead to accidents. In a multinational study, Moran et al. tested 373 physical education students [18]. The students first provided self-estimates of water competence (via a questionnaire) and then performed six practical tests in a swimming pool. The results showed a significant correlation between stated and actual competence for tests at the surface of the water (swimming for as long as possible during a 15-minute period, floating as long as possible without moving, and a $100 \mathrm{~m}$ backstroke test). The dangerousness of the underwater tasks prompted the 
researchers to take a number of precautions. Sixty-two percent of the students answered "yes" to the question "Can you swim underwater?". In the pool, there was no minimum distance requirement for underwater swimming (for obvious safety reasons). This made it difficult to measure the motor competence with respect to the question "Can you swim underwater". The researchers failed to observe a correlation between perceived and real competence in this context (Spearman's rank correlation coefficient $r=-0.134$ ) because only $37 \%$ of the students swam more than 25 $\mathrm{m}$ underwater. Were these the only students to have validated the competency of the underwater experiment? It is questionable whether this finding confirmed the young adults' tendency to overestimate their motor competence in an aquatic milieu.

The present study was designed to measure subaquatic motor competence in this type of population (physical education students), while removing the risk associated with the imposition of a minimum distance or time (as is usually the case for tests at the surface) but also while blinding the participants to the study's objective. To this end, we studied a pure coordination game based on the work of Schelling [19]. The swimmers' goal was to surface at the same distance as an unknown partner of the same ability, rather than to push themselves as far as possible and equal their prior (over) estimation.

In contrast to literature studies of young men's overestimation of water competence in a challenging situation, the present study's starting hypothesis was that the implementation of a coordination game would reduce overestimation. Since the swimmers did not have to achieve a maximal performance, they would do better than they had previously imagined.

\section{Experimental Design}

A game invented by the Thomas Schelling (winner of the 2005 Nobel Prize in Economics) was adapted for use in the present experiment. Thomas Schelling (winner of the 2005 Nobel Prize in Economics) asked people to imagine that they had to meet someone in
New York on a specific day but without knowing the time or place of the meeting and without being able to communicate with the person in question. An overwhelming majority of the respondents chose Grand Central Station as the meeting place and midday as the meeting time. These responses prompted Schelling to introduce the notion of a "focal point" (today referred to as the "Schelling point"). From among thousands of Nash equilibria, the influences of culture, personal preference and empathy mean that a single Nash equilibrium emerges.

In this two-player pure coordination game, the latent solution is based on the presumption of reciprocity [20, 21]: "I will choose this because I think that the other person will choose it, and I think that the other person will choose it because he/she thinks that I will choose it". The game's solution cannot be rationally calculated; in many cases, there is an almost infinite number of Nash equilibria [22], no maximin solution [23] and no subordinating moral value [24]. Tacit coordination is based subjectively on the estimation of an obvious focal point. There are many illustrations of Schelling's point [19]: (1) when asked to choose "heads or tails" (and knowing that the same choice as a partner counts as a win), 36 people chose "tails" and 6 choose "heads"; (2) when told "write down an amount of money: if your partner writes down the same amount, you will win that amount", 12 out of 41 people chose 1 million dollars and only three answers concern figures other than powers of 10; (3) two parachutists have landed on a same area but are not in sight of each other. Each knows that the other has the same map but neither knows his/her exact position and no means of communication are available. However, in order to complete their mission, the two parachutists have to find each other. When the map shows the region's topography clearly, experience shows that most of the pairs of parachutists end up by finding each other.

\subsection{Procedure}

How then would swimmers (rather than parachutists) 
play this type of game in a $25 \mathrm{~m}$ swimming pool? One could hypothesize that the swimmers will agree on the place at which they expect to meet their partner. Initially, swimmers had to answer a paper self-questionnaire with the following scenario and main question: "You are playing separately with someone with whom you cannot communicate. You start by diving into a swimming pool and you may only swim up the lane underwater. At what distance from the start are you going to surface (knowing that to win, you have to do surface at exactly the same point as your partner)? Your (unknown) partner has exactly the same information as you and will play without having seen you swim. To win this game, where should you surface?"

Next, each swimmer in turn dived into the pool and had to surface at the "meeting point"-the SP ("Schelling point") chosen by a partner whom he had not met. There were no distinctive markings on the bottom of the pool or elsewhere in the field of vision. During the game, the other swimmers waited with their backs to the pool and were not allowed to communicate with the other players. The participating students were told that their grade for the swimming course would be raised by one point if they won. At the end of the game, each swimmer's maximum underwater swimming distance was tested (but without any element of competition). The results in the Schelling game were compared with the maximum potential distance by calculation of Spearman's rank correlation coefficient. The distance swum underwater was measured from the surface with respect to the lane distance markers (at one-meter intervals). The value was rounded to the nearest half-meter.

\subsection{Study Participants}

The group of swimmers was composed of 43 male university sports students aged between 18 and 20 . The participants were moderately able swimmers (Table 1). All had given their informed consent to voluntary participation in the Schelling game. The swimming experiments formed part of their university course and the participants were insured by the host university. Thirty-eight students from the same university course served as controls (Table 1): they filled out the questionnaire but were told that they would not have to test their estimation in the pool.

Prior to the test, participants were provided with comprehensive information about the study procedures. All gave their written informed consent to participation, in accordance with the tenets of the Declaration of Helsinki (1975).

\subsection{Measures}

(1) $12.5 \mathrm{~m}$ (half the length of the pool, i.e. the distance equivalent of "noon") should correspond to the SP predicted by theory, with a normal (Gaussian) distribution around this distance. We expected that the interventional group would reply in the same way as the control group (as measured in a Student's T test), even though members of the control group knew that they would not have to put their decision into practice. In the pool, the further the participants swim beyond this SP, the more they would have underestimated their underwater swimming ability and that of their (unknown) partners.

(2) If the true SP for motor action is greater than the theoretical SP (12.5 m), the group will feel at ease with underwater swimming ("we can go farther under water than we expected").

(3) In contrast, if the true SP distance is shorter than the theoretical SP, then the swimmers will have been inhibited by the aquatic environment and expected to swim further than they really could.

Our secondary hypothesis was that: (4) That there

Table 1 Characteristics of the study population.

\begin{tabular}{ll}
\hline The test group (swimmers) & The control group \\
\hline 43 male physical education & 38 male physical education \\
students (aged 18-20) from & students (aged 18-20) from \\
Paris Descartes University & Paris Descartes University \\
(Paris, France). & (Paris, France). \\
\hline $\begin{array}{l}\text { Swimming ability: able to swim } 420 \mathrm{~m} \text { in } 10 \text { min and to swim } \\
\text { underwater for at least } 8 \mathrm{~m} .\end{array}$ \\
\hline
\end{tabular}


was a correlation between the player's maximum underwater swimming distance and the way in which he played this pure coordination game (as measured by Spearman's correlation coefficient $r$ ).

\section{Results}

The experimental data validated hypotheses (1) and (2) but not hypothesis (3). The median distance stated on the pre-swim questionnaire was $12.5 \mathrm{~m}$. This value was given by 14 of the 43 interventional group members (33\%) and 12 of the 38 students in the control group. A random frequency would yielded a value of 43 (or 38) divided by the 26 possible one-metre zones (with 0 to 2 swimmers per zone). This was indeed the SP predicted by theory (Figs. 1 and 2). However, in the water, the swimmers behaved differently. They always broke the surface further away than they expected and the SP was $25 \mathrm{~m}$ - the greatest possible distance. Fifteen of the 43 swimmers swam this distance and 26 others surfaced after more than $12.5 \mathrm{~m}$ (Fig. 3). The

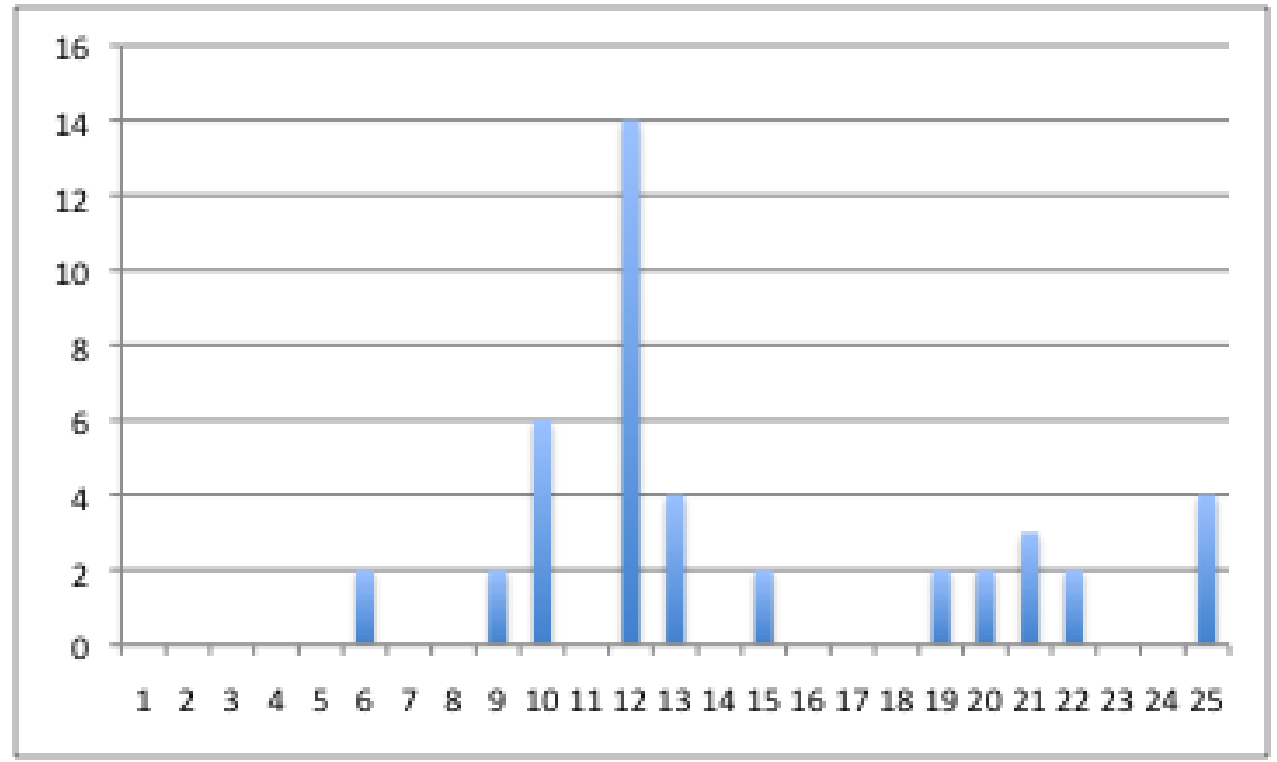

Fig. 1 Distribution of the breakout (surfacing) distances in the SP swimming game, as stated on the pre-swim questionnaire by the swimming group $(n=43)$. The $x$ axis shows the distance in one-metre zones. The $y$ axis shows the number of answers per zone. On the questionnaire, the most frequent response given by the 43 swimmers was $12.5 \mathrm{~m}$ (rounded down to $12 \mathrm{~m}$ on the graph: this is the SP).

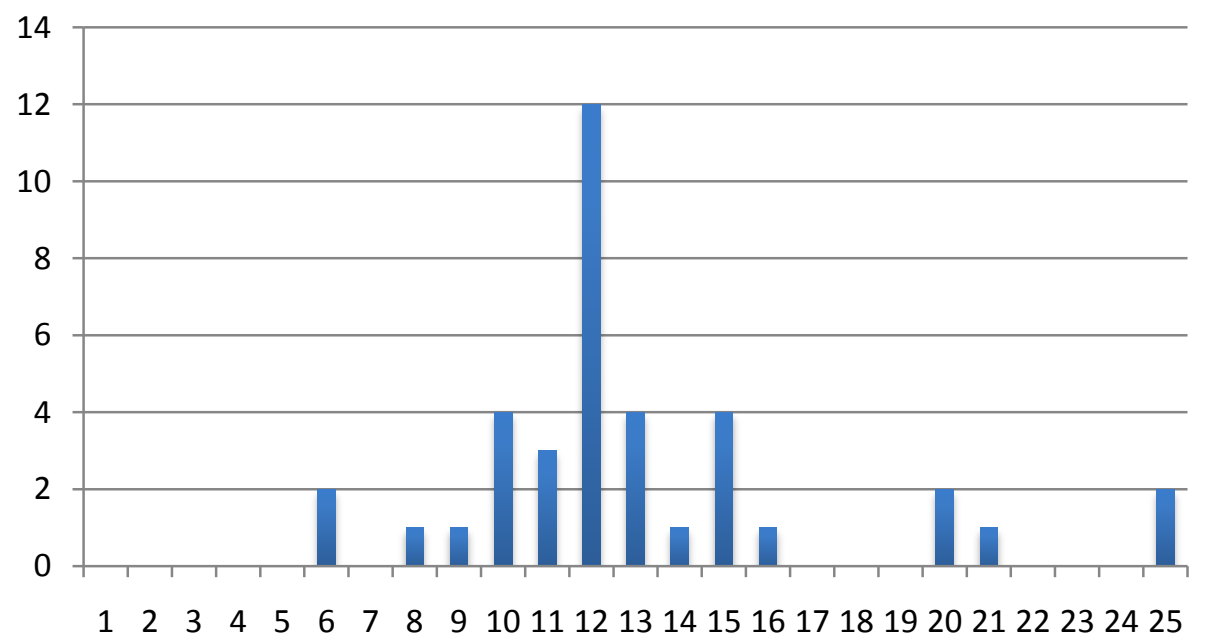

Fig. 2 Distribution of the breakout distances in the SP swimming game, as stated on the questionnaire by the control group (n = 38). As in the interventional group, the middle of the pool $(12.5 \mathrm{~m})$ was designated most frequently as the focal point. 


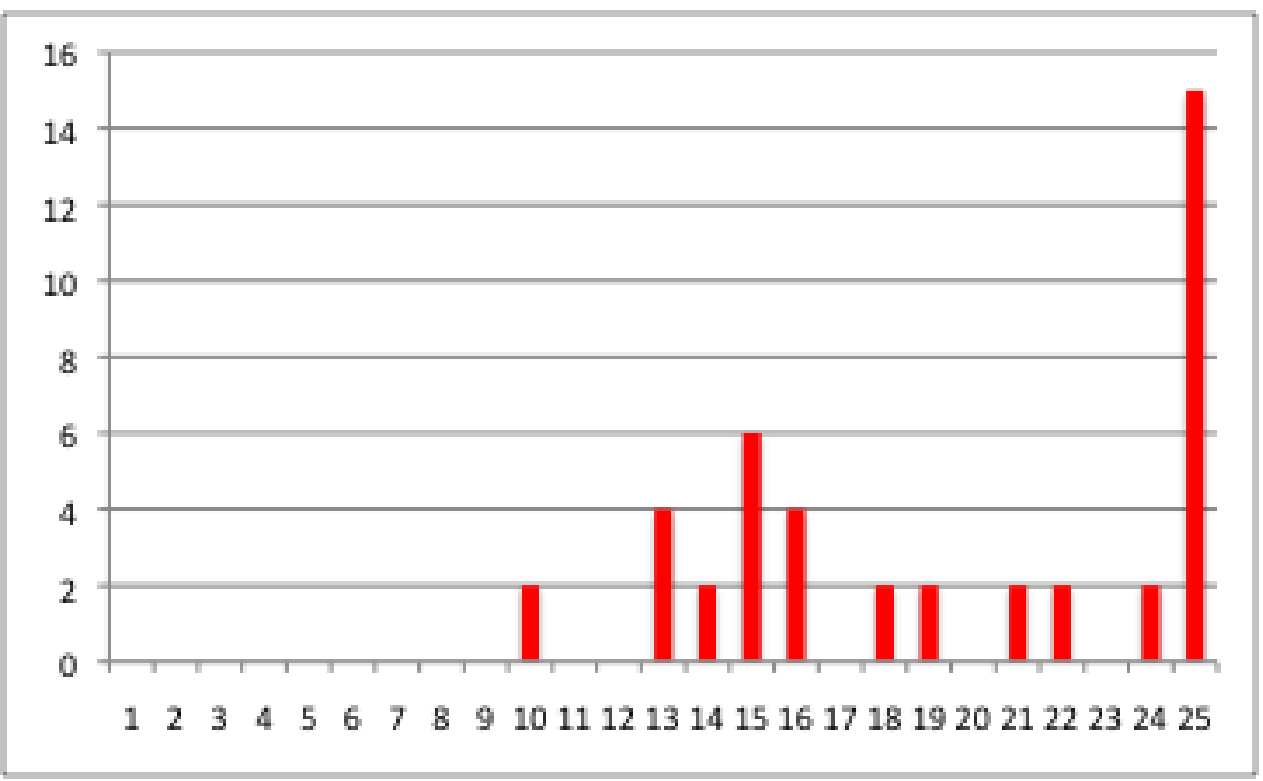

Fig. 3 Distribution of the breakout distances in the SP swimming game; as performed in the pool by the interventional group $(n=43)$. In the water, the swimmers surfaced most often at $25 \mathrm{~m}$; this second focal point (SP2) corresponds to the maximum distance that could be swum underwater in this game.

presence of two SPs is an unusual experimental feature. The SP of 12.5 (SP1) corresponded to the distance expected for this type of game. The second, "illogical" SP achieved in practice (SP2) testified to (1) a marked disparity between what was had been stated and what was actually done, suggesting that the characteristics of the test environmental perturbed the participants' ability to fulfil their estimated potential and (2) marked enjoyment of underwater swimming and the belief that the swimmer's unknown partner would do the same. After the experiment, we recorded three types of feedback. Firstly, some participants said that they had been unable to locate their anticipated surfacing point (because of the lack of markings on the pool bottom) and were surprised to have surfaced so far out. Secondly, some participants "felt good" underwater and swum further because they presumed the others would feel the same way. Thirdly, some participants thought that given the lack of markings on the pool bottom, the only way to find a focal point was to swim underwater to the end of the pool. In all cases, this pure coordination game did not incite swimmers to behave competitively through swimming as far as possible underwater. The other visible point in the pool (apart from the far end) was the starting wall (i.e. distance $=0$ $\mathrm{m})$ but none of the swimmers stopped there-even though they could have done so by jumping vertically into the pool and resurfacing immediately. In contrast to preconceived ideas (and in a non-obligatory context), the participants (who were not competitive swimmers and were merely able to swim $420 \mathrm{~m}$ in 10 minutes) appeared to simply like holding their breath for a long time.

As in the interventional group, the middle of the pool $(12.5 \mathrm{~m})$ was designated most frequently as the focal point. In the water, the swimmers surfaced most often at $25 \mathrm{~m}$; this second focal point (SP2) corresponds to the maximum distance that could be swum underwater in this game.

The secondary hypothesis was not validated. There was a correlation between the estimated distance swum underwater (the SP) and the participant's maximum underwater swimming ability (as assessed a week later; ( $\mathrm{r}=+0.56, P<0.01$, Table 2 - Spearman's rank correlation coefficient is between -1 and +1 ). The greater the distance the swimmers could cover in the test, the longer they stayed underwater during the SP game. 
Table 2 Spearman rank correlations calculated for ordinal variables (distances in the SP game in the pool (SP by Action), distances in the SP game stated on the questionnaires (SP by Question) and maximum underwater swimming distances (Max underwater).

\begin{tabular}{llll}
\hline$>\mathrm{N}=43$ & SP-Action & SP-Question & $\begin{array}{l}\text { Max } \\
\text { underwater }\end{array}$ \\
SP-Action & & $\mathrm{r}=+0.34$ & $\mathrm{r}=+0.56$ \\
& & $P<0.05$ & $P<0.01$ \\
SP -Question & & & $\mathrm{r}=-0.17$ \\
Max & & $N S$ \\
underwater & & & \\
\hline
\end{tabular}

\section{Discussion}

When faced with a challenge, young adults tend to overestimate their water competence [14-16, 18].Our results suggest that this is not the case when participants play a pure coordination game (such as the SP game). The goal was to swim as far underwater as an unknown, invisible partner would, rather than to achieve or exceed a previous estimation. In the absence of competitive pressure, the participants appeared to be more at ease under the water. Lastly, the swimmers performed better than they expected to. It is noteworthy that the mean distance swum underwater in the present study was $19.3 \mathrm{~m}$, which is distinctly greater than the distance of $17.8 \mathrm{~m}$ recorded for the 373 swimmers in the multinational study by Moran et al. [18] (in which the context was "Can you swim underwater?"). The SP experiment in the pool did not restrict water competence and enabled the participants to show their true abilities with no outside pressure. This finding is encouraging and suggests that use of this type of game in other areas of physical education would reduce fear of water and other behaviors associated with risk.

The results show (1) a marked disparity between what was had been stated and what was actually done, suggesting that the characteristics of the test environmental perturbed the participants' ability to fulfil their estimated potential and (2) marked enjoyment of underwater swimming and the belief that the swimmer's unknown partner would do the same. After the experiment, we recorded three types of feedback. Firstly, some participants said that they had been unable to locate their anticipated surfacing point (because of the lack of markings on the pool bottom) and were surprised to have surfaced so far out. Secondly, some participants "felt good" underwater and swum further because they presumed the others would feel the same way. Thirdly, some participants thought that given the lack of markings on the pool bottom, the only way to find a focal point was to swim underwater to the end of the pool. In all cases, this pure coordination game did not incite swimmers to behave competitively through swimming as far as possible underwater. The other visible point in the pool (apart from the far end) was the starting wall (i.e. distance $=0$ $\mathrm{m})$ but none of the swimmers stopped there-even though they could have done so by jumping vertically into the pool and resurfacing immediately. In contrast to preconceived ideas (and in a non-obligatory context), the participants (who were not competitive swimmers and were merely able to swim $420 \mathrm{~m}$ in $10 \mathrm{~min}$ ) appeared to simply like holding their breath for a long time.

The present results must be confirmed in other populations, and the influence of gender, age and ability in other coordination games (notably Binmore's "ultimatum game" [25]) must be investigated. Indeed, players may behave differently when subjected to other rules (even when the latter are cooperative); the nature of the game has a great influence on the player. We conclude that game theory is a promising tool for comparing perceived and real motor skills.

\section{References}

[1] Rudisill, M. E., Mahar, M. T., and Meaney, K. S. 1993. "The Relationship between Children's Perceived and Actual Motor Competence." Perceptual \& Motor Skills 76 (3 Pt 1): 895-906.

[2] Raudsepp, L., and Liblik, R. 2002. "Relationship of Perceived and Actual Motor Competence in Children." Perceptual \& Motor Skills 94 (3 Pt 2): 1059-70.

[3] Sollerhed, A. C., Apitzsch, E., Råstam, L., and Ejlertsson, G. 2008. "Factors Associated with Young Children's Self-Perceived Physical Competence and Self-Report Physical Activity." Health Education Research 23 (1): 
125-36.

[4] Moran, K. 2011. "Perceived and Real Swimming Competence among Young Adults in New Zealand." In Proceedings of the World Drowning Prevention Conference, Da Nang, Vietnam.

[5] Wang, J., Liu, W., and Bian, W. 2013. "Relationship between Perceived and Actual Motor Competence among College Students." Perceptual \& Motor Skills 116 (1): 272-9.

[6] Counsilman, J. E. 1977. Competitive Swimming Manual for Coaches and Swimmers. Bloomington: Counsilman Company.

[7] Collard, L., Oboeuf, A., and Ahmaidi, S. 2007. "Motor Skills Transfer from Gymnastics to Swimming." Perceptual and Motor Skills 105 (1): 15-26.

[8] Howland, J., Hingson, R., Mangione, T. W., Bell, N., and Bak, S. M. P. H. 1996. "Why are Most Drowning Victims Men? Sex Differences in Aquatic Skills and Behaviors." American Journal of Public Health 86 (1): 93-6.

[9] Gilchrist, J., Sacks, J. J., and Branche, C. M. 2000. "Self-Reported Swimming Ability in US Adults." Public Health Reports 115 (2-3): 110.

[10] Gulliver, P., and Begg, D. 2005. "Usual Water Related Behaviour and 'Near Drowning' Incidents in Young Adults." Australian and New Zealand Journal of Public Health 29 (3): 238-43.

[11] Moran, K. 2006. "Re-thinking Drowning Risk: the Role of Water Safety Knowledge, Attitudes and Behaviors in Aquatic Recreaction of New Zealand Youth.” Ph.D. thesis, Palmerston North: Massey University, New Zealand.

[12] McCool, J. P., Moran, K., Ameratunga, S., and Robinson, E. 2008. "New Zealand Beachgoers' Swimming Behaviors, Swimming Abilities, and Perception of Drowning Risk". International Journal of Aquatic Research \& Education 2 (1): 7-15.

[13] Rahman, A., Rahman, F., Mashreky, S., and Linnan, M. 2011. "A Longitudinal Study of Child Drowning in Rural
Bangladesh." Presented at the Proceedings of the World Drowning Prevention Conference, Da Nang, Vietnam.

[14] Baker, S. P., O’Neil, B., Ginsburg, M. J., and Li, G. 1992. The Injury Fact Book. New York: Oxford University Press.

[15] Fisher, J. 1981. "Reduce Young Children's Fear of Water." Journal of Physical Education and Recreation 52 (4): 11.

[16] Stillwell, B. 2011. "Best Practices for Teaching Those Afraid in Water." US-China Education Review 8 (2): 249-55.

[17] Bay-Hinitz, A. K., Peterson, R. F., and Quilitch, H. R. 1994. "Cooperative Games: A Way to Modify Aggressive and Cooperative Behaviors in Young Children." Journal of Applied Behavior Analysis 27 (3): 435-46.

[18] Moran, K., Stallman, R. K., Kjendlie, P. L., Dahl, D., Blitvich, J. D., Petrass, L. A., and Shimongata, S. 2012. "Can You Swim? An Exploration of Measuring Real and Perceived Water Competency." International Journal of Aquatic Research \& Education 6 (2): 122-35.

[19] Schelling, T. 1960. The Strategy of Conflict. Harvard University Press.

[20] Crawford, V. P., and Haller, H. 1990. "Learning How to Cooperate: Optimal Play in Repeated Coordination Games." Econometrica, Econometric Society 58 (3): 571-95.

[21] Sugden, R. 1995. "A Theory of Focal Points.” Economic Journal, Royal Economic Society 105 (430): 533-50.

[22] Nash, J. 1950. "Equilibrium Points in $n$-Person Games". In Proceedings of the National Academy of Sciences of the USA 36: 48-9.

[23] Von Neumann, J., and Morgenstern, O. 1944. Theory of Games and Economic Behavior. Princeton: Princeton University Press.

[24] Harsanyi, J. 1977. "Morality and the Theory of Rational Behaviour." Social Research 44 (4): 623-56.

[25] Binmore, K. 1992. Fun and Games: A Text on Game Theory. Heath and Company: Lexington, Massachusetts. 